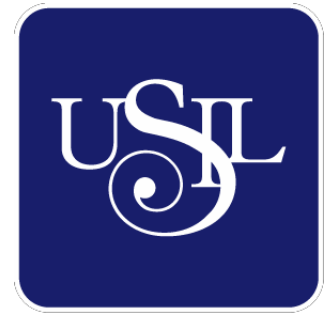

UNIVERSIDAD

SAN IGNACIO

DE LOYOLA

ESCUELA DE POSTGRADO

Maestría en Administración de Negocios (MBA)

\title{
PLAN ESTRATÉGICO DE LA EMPRESA VIVALO IMPORT EXPORT E.I.R.L., LIMA, PARA EL PERIODO $2019-2022$
}

Trabajo de Investigación para optar el grado de Maestro en Administración de Negocios (MBA)

KENLY AUGUSTO VALENTÍN CAJAS

LUIS ALBERTO ABAD MARTÍNEZ

Asesor:

Mg. Flor Elvira Rios Rivero

Lima - Perú

2019 
A Dios

Por brindarnos la fuerza para lograr nuestro gran objetivo.

A nuestra familia Por brindarnos todo el apoyo y paciencia. 


\section{Resumen Ejecutivo}

El presente Plan Estratégico elaborado para la empresa Vivalo Import Export E.I.R.L. (Editorial Berlín) para el periodo 2019 - 2022, cuya actividad está basada en la producción y comercialización de coleccionables impresos (álbumes, figuritas y cards), propondrá estrategias que le permitirán seguir creciendo e incrementar su rentabilidad.

Durante la investigación se ha encontrado el potencial y futuro que tiene la comercialización de álbumes y figuritas en el entretenimiento y en el aprendizaje de los niños, que fue reimpulsada por la participación de la selección peruana en el Mundial Rusia 2018. En este aspecto, el impacto que tienen los coleccionables garantiza su permanencia en el tiempo, generando, además, que las costumbres se transmitan de padres a hijos y de estos a sus hijos. Los coleccionables permiten que los niños interactúen durante el llenado de los álbumes, la revisión de los faltantes y del pegado de las figuritas, asimismo, les ayuda en su aprendizaje de las transacciones que deben realizar durante el intercambio de figuritas con sus compañeros.

Los expertos en psicología infantil ofrecen buenas razones que avalan el impacto positivo del álbum de figuritas en los niños, i) como la acción de coleccionar figuritas y pegar en el álbum ayuda a los niños a memorizar números o imágenes, facilitando y ejercitando la concentración, ii) mejora los vínculos familiares, teniendo en cuenta que durante el proceso de llenar el álbum, los niños son asistidos por los padres, abuelos, tíos o hermanos más grandes y iii) los niños aprenden a intercambiar con sus pares sus "figuritas repetidas" por las que aún no tienen, deben hacer valer las suyas y en caso de no querer hacer trato, poder decir que no, se pueden equivocar y arrepentirse del trato hecho, que aprenderán de la situación para la próxima (Inter-cole-en-red, 2018).

Las estrategias propuestas en el presente plan comprenden la penetración de mercado y desarrollo de producto con la finalidad de que la empresa Vivalo Import Export E.I.R.L. tenga mayor presencia en el mercado e incremente sus utilidades.

La inversión en el presente Plan Estratégico es en promedio de S/ 255,465 por año, que le permitirá una mayor expansión de las ventas a finales del año 2022 (S/ 7,190,500 ), un retorno de la inversión del propietario de S/ 1,187,200, con un costo de capital de $16.6 \%$. 


\section{Tabla de Contenido}

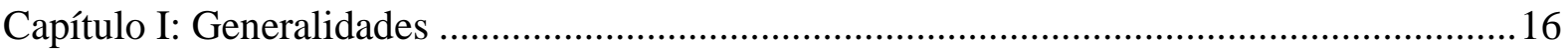

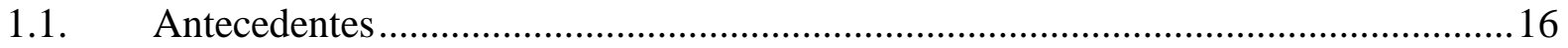

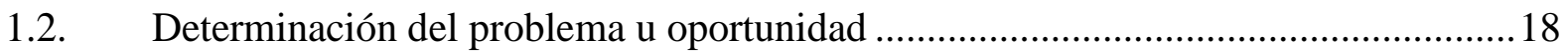

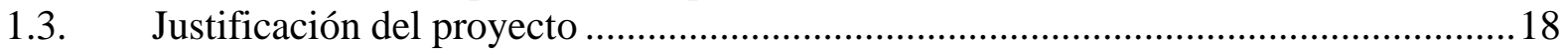

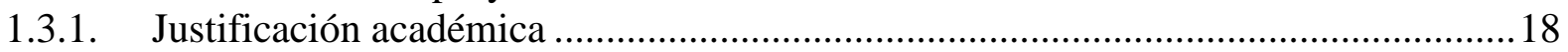

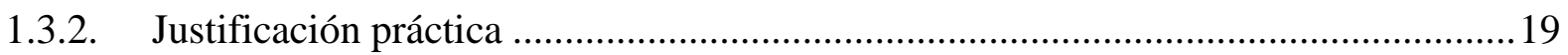

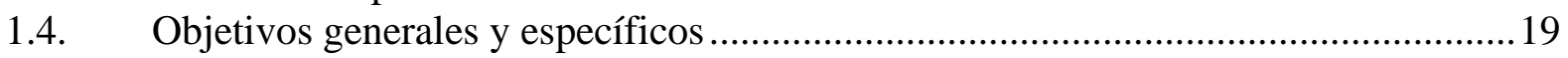

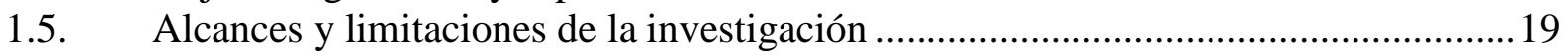

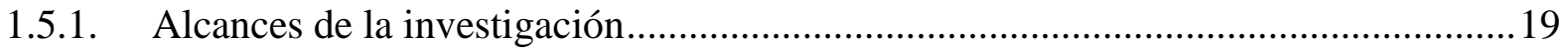

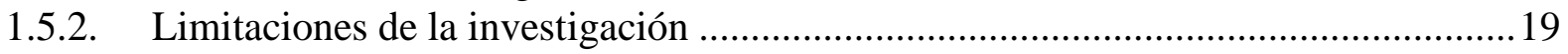

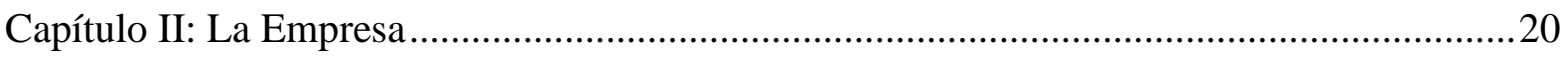

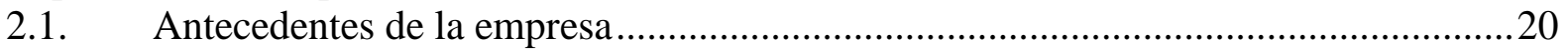

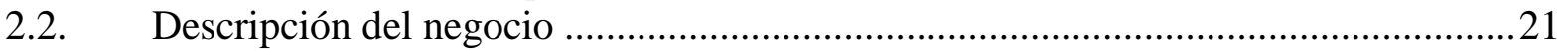

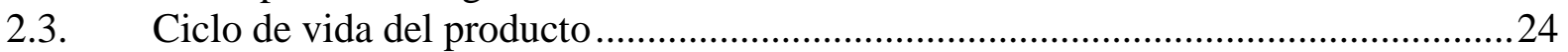

2.4. Estructura organizacional actual de la empresa.....................................................26

2.5. Situación de Mercado y Financiera actual de la Industria ........................................28

Capítulo III: Formulación de Visión, Misión y Valores de la Empresa ...................................32

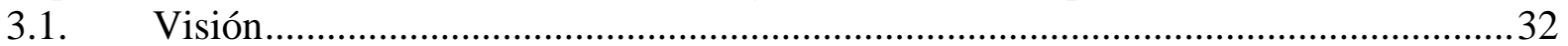

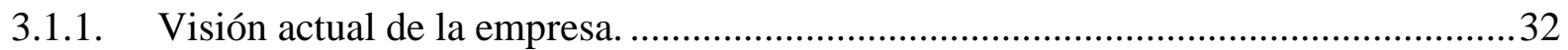

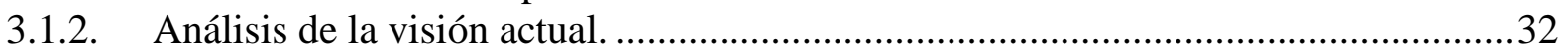

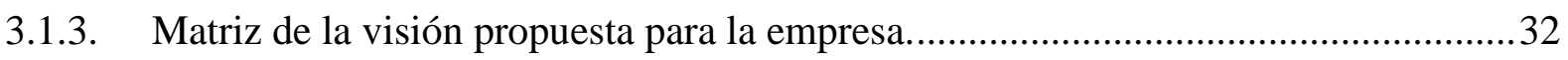

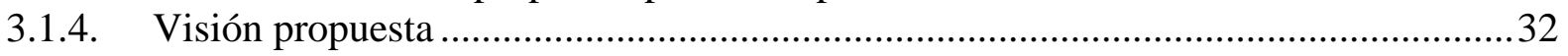

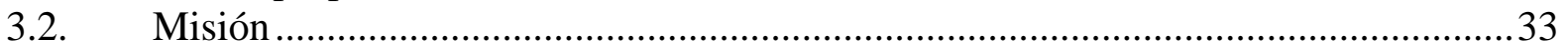

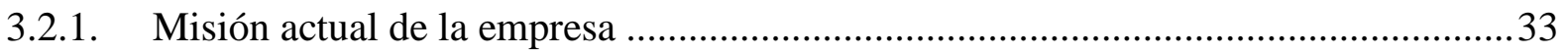

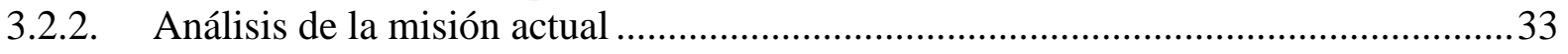

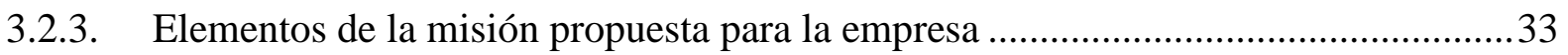

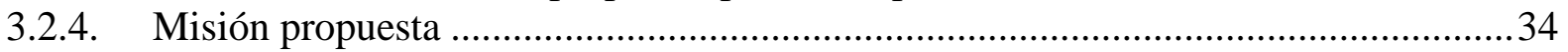

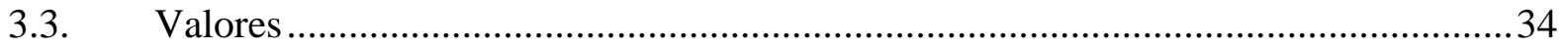

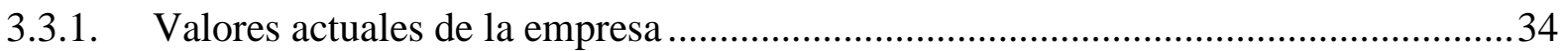

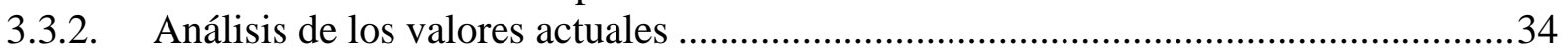

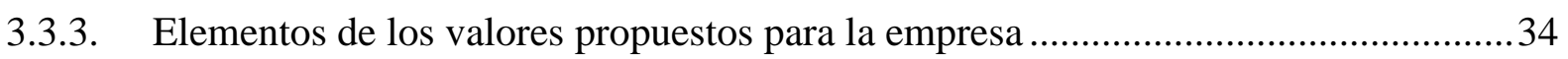

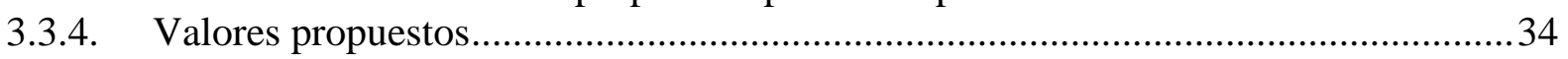

3.4. Alineamiento estratégico de la Visión, Misión y Valores de la empresa ....................36

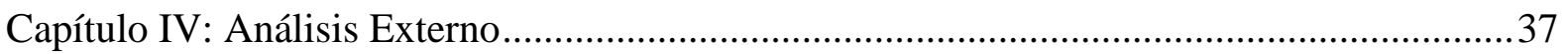

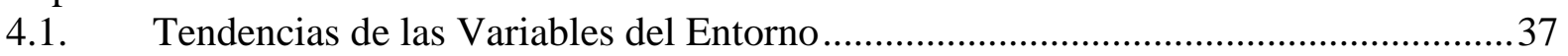

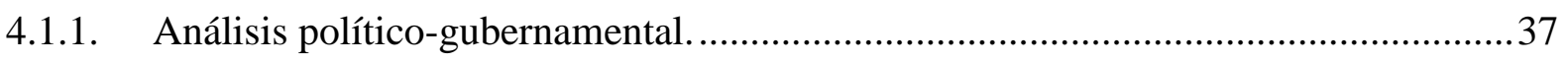

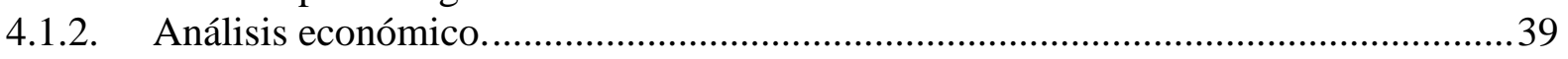

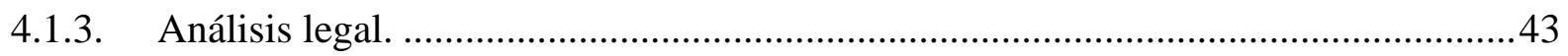

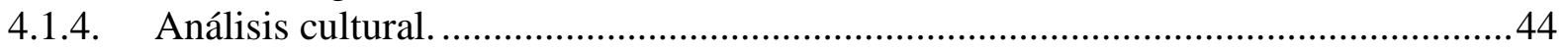

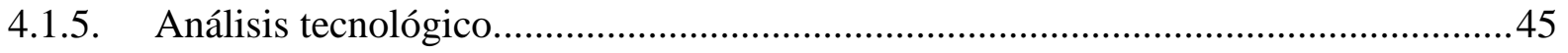

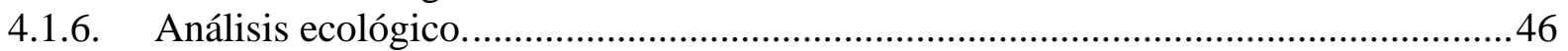

4.2. Impacto en Clientes / Proveedores de cada una de las Variables del Entorno ..........47

4.3. Efecto en la Empresa de cada una de las Variables del Entorno ...............................48

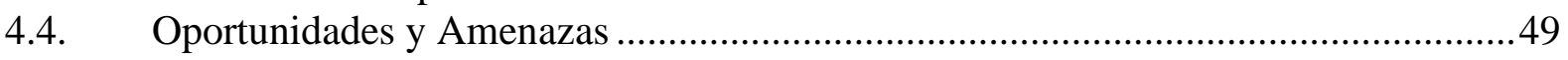




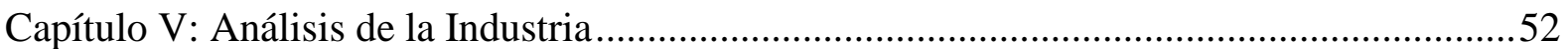

5.1. Descripción del Mercado (demanda) e Industria (oferta) .........................................52

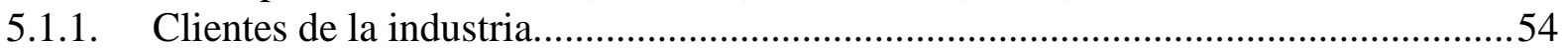

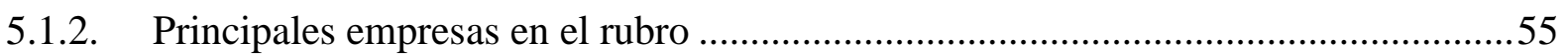

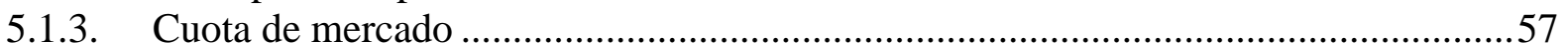

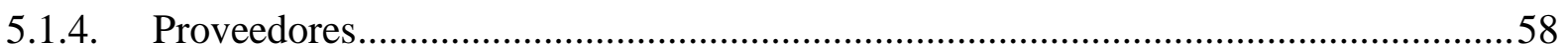

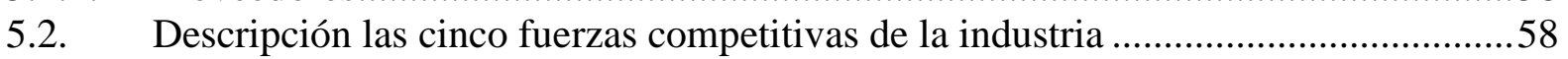

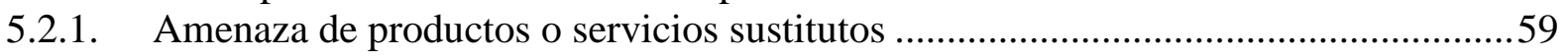

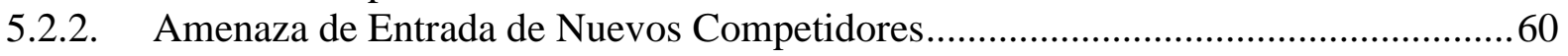

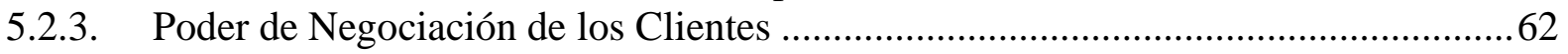

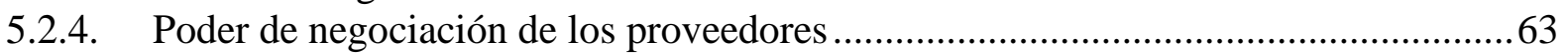

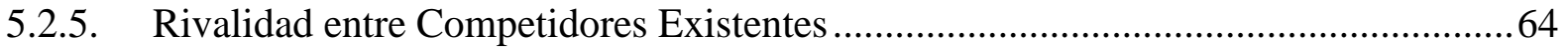

5.3. Matriz de atractividad de cada una de las cinco fuerzas.........................................66

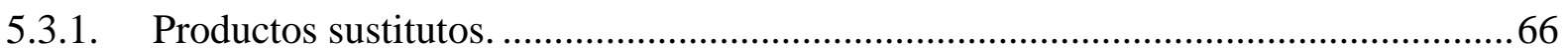

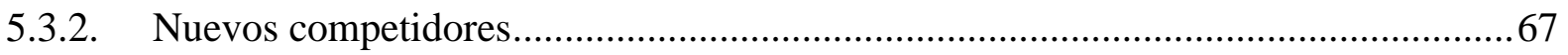

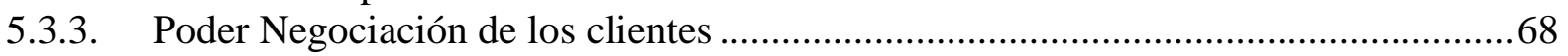

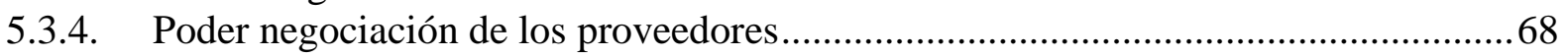

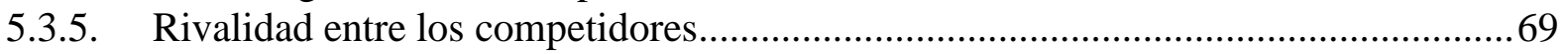

5.4. Análisis del Grado de atractividad de la industria ..................................................... 70

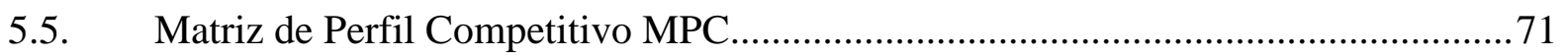

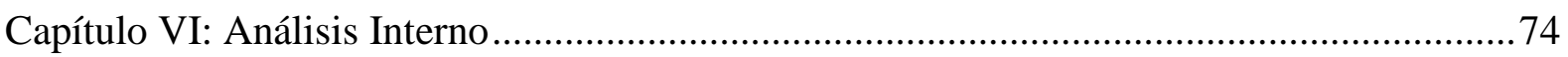

6.1. Descripción de las actividades de la cadena de valor de la empresa. ........................74

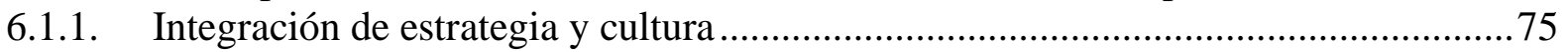

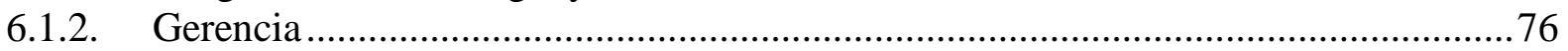

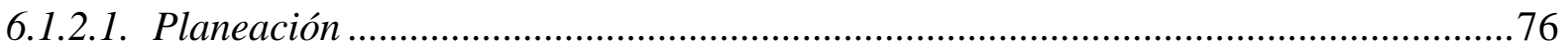

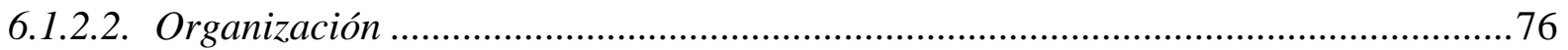

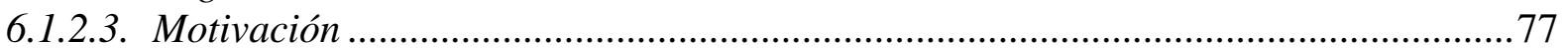

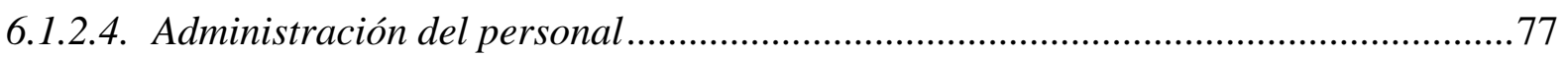

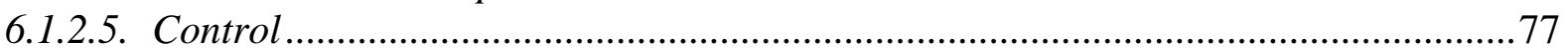

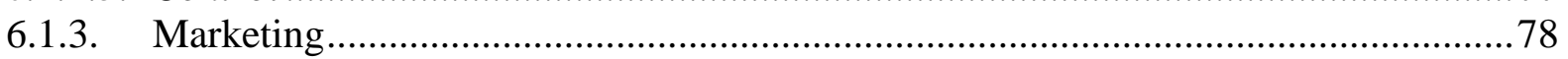

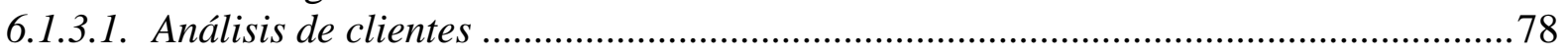

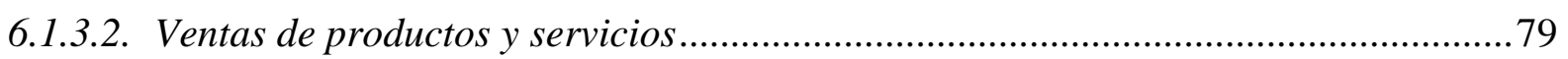

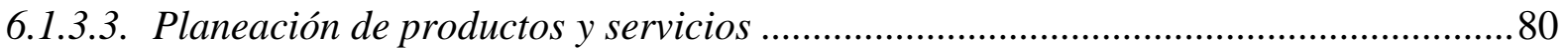

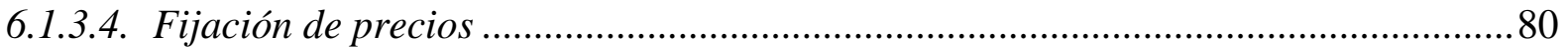

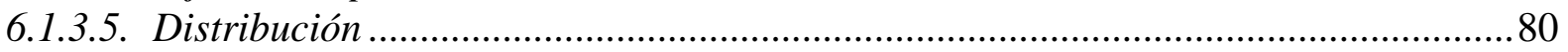

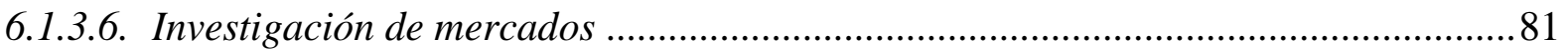

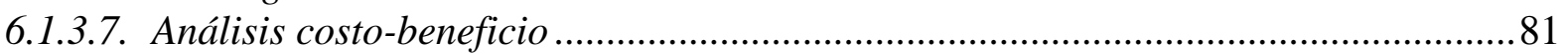

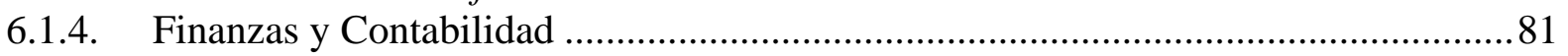

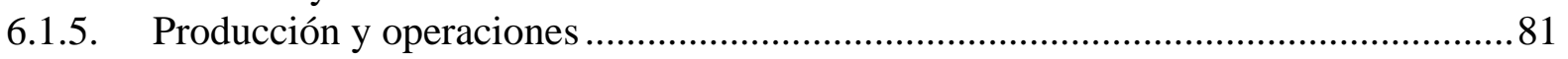

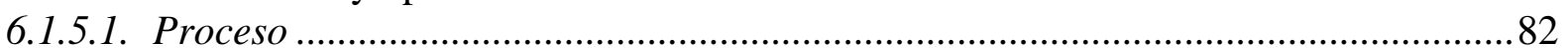

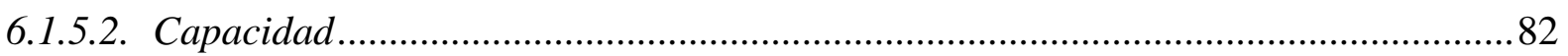

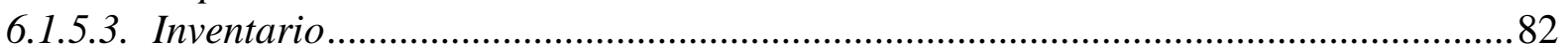

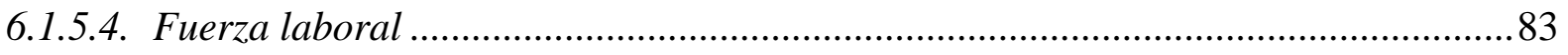

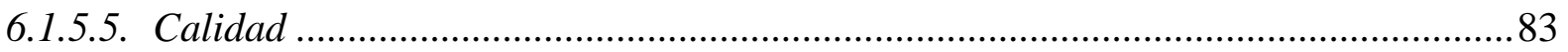

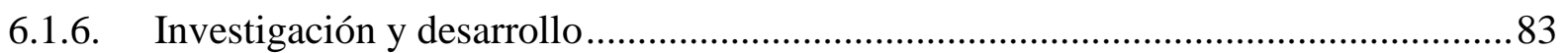

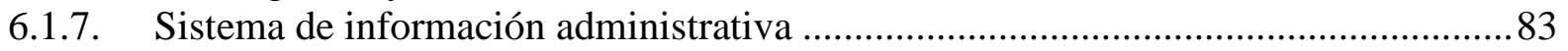




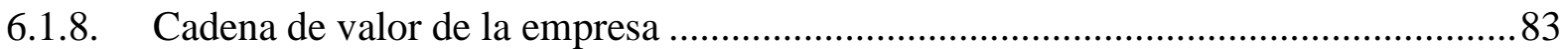

6.2. Indicadores de cada una de las actividades de la cadena de valor ............................87

6.3. Benchmarking y comparación con los líderes de la industria de cada una de las

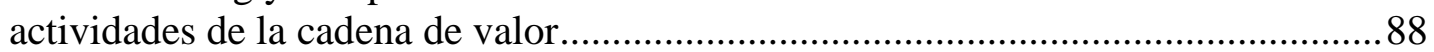

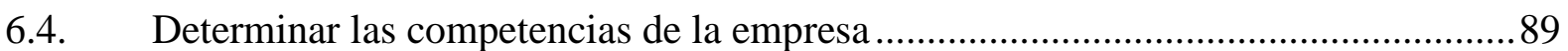

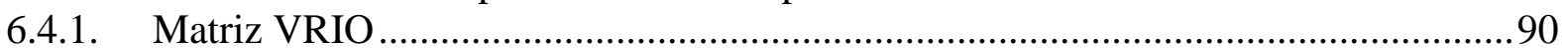

6.5. Identificación y determinación de las ventajas competitivas de la empresa .............91

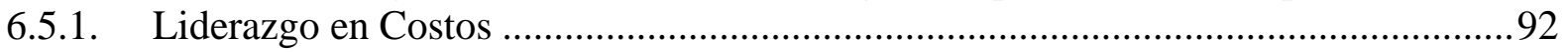

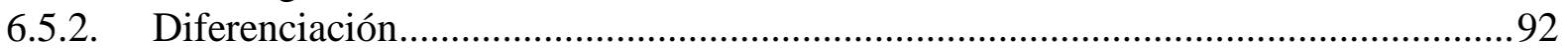

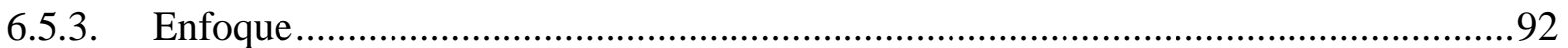

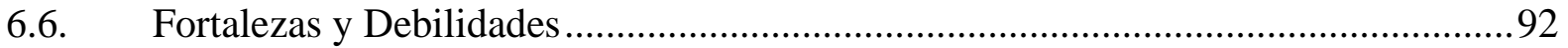

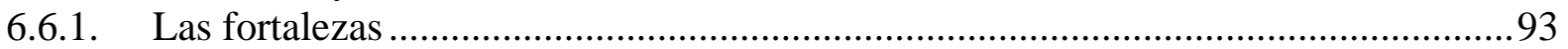

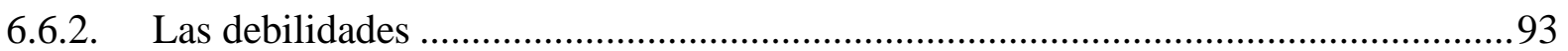

6.7. Matriz de Evaluación de los Factores Internos EFI................................................95

Capítulo VII: Formulación de los Objetivos y Diseño de las Estrategias...............................96

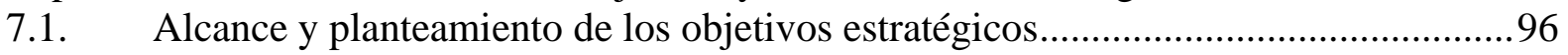

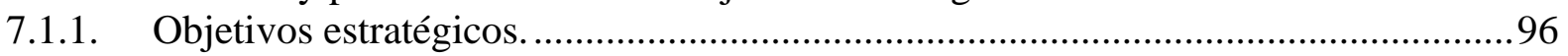

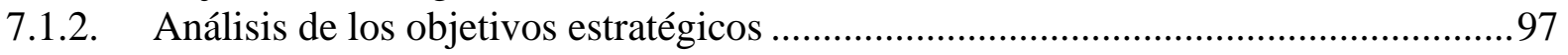

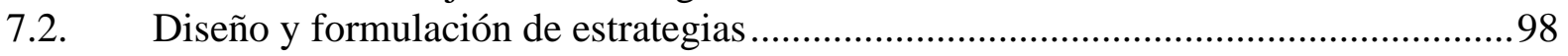

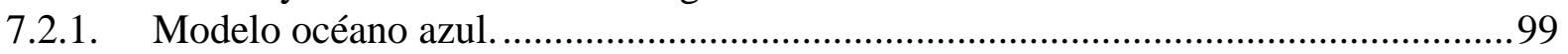

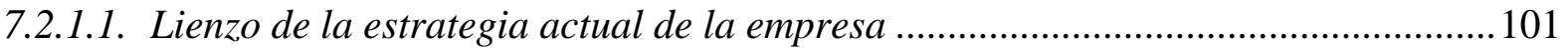

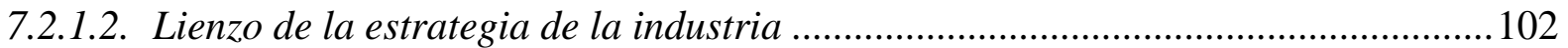

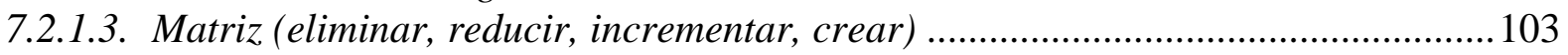

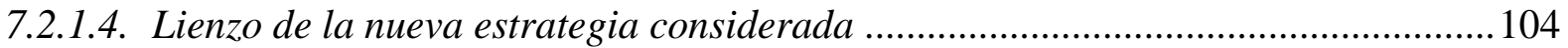

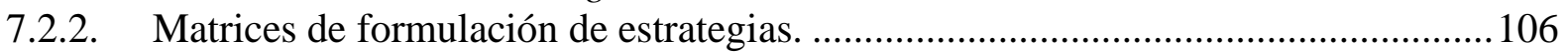

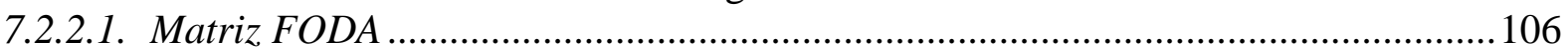

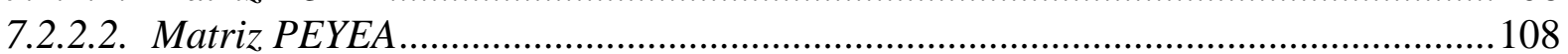

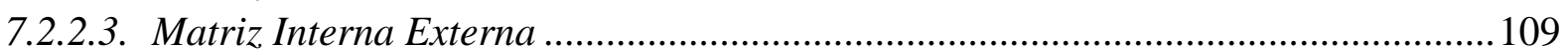

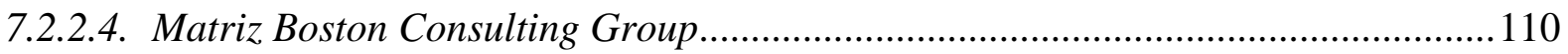

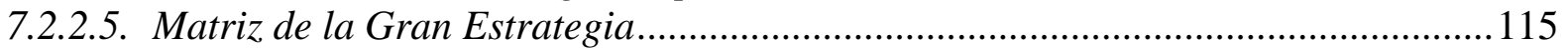

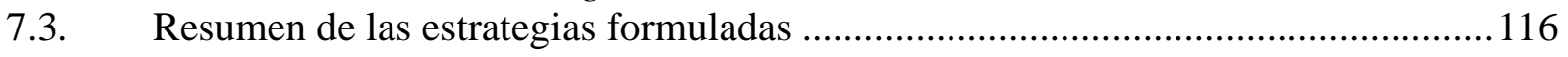

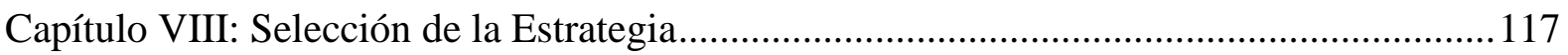

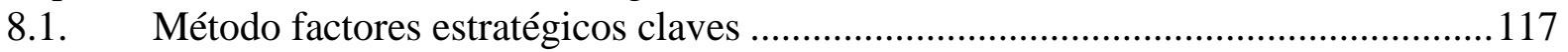

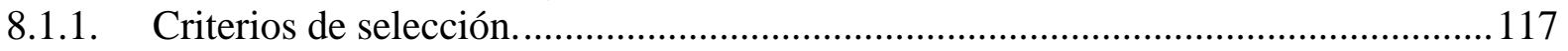

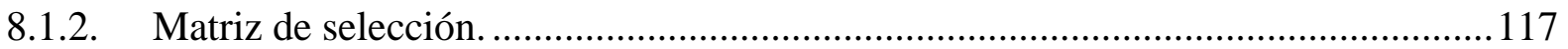

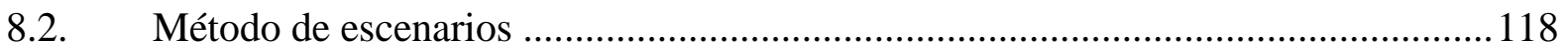

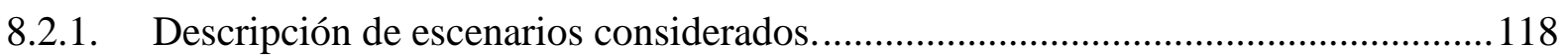

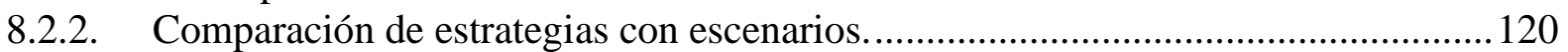

8.3. Matriz de Planeación Estratégica Cuantitativa - MPEC ........................................... 121

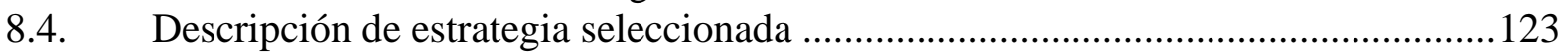

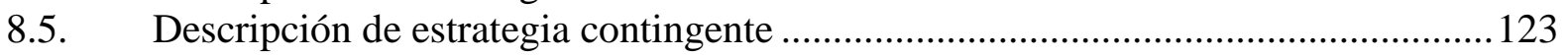

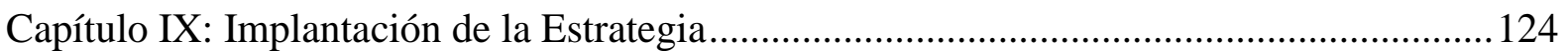

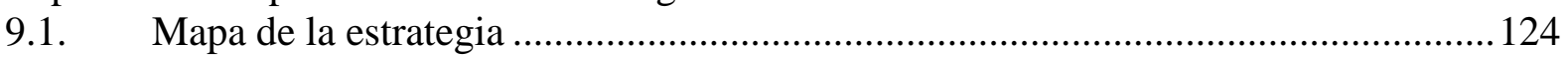

9.2. Objetivos específicos según el mapa de la estrategia ............................................ 125

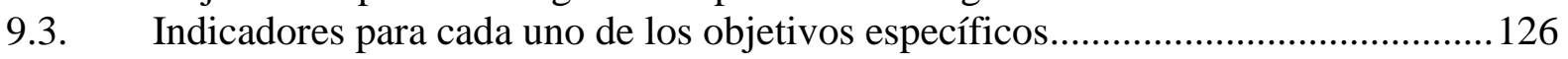

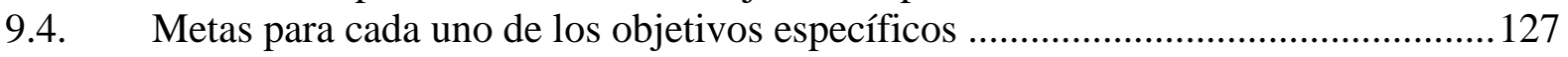


9.5. Iniciativas (acciones a llevar a cabo para cada uno de los objetivos específicos) estrategias, programas, políticas, reglas, procedimiento

9.6. Responsable de cada una de las iniciativas............................................................ 128

9.7. Matriz de objetivos, indicadores e iniciativas de las estrategias ............................ 128

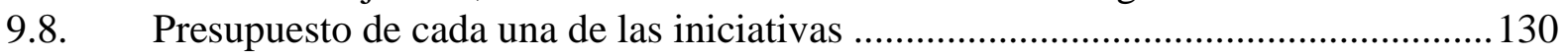

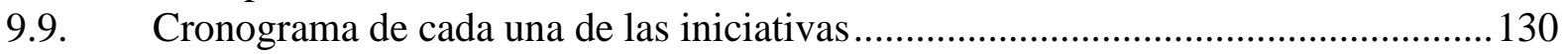

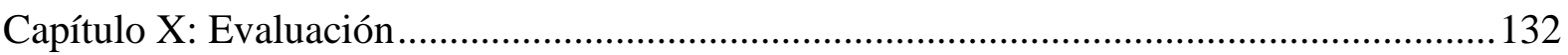

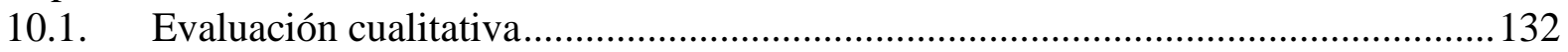

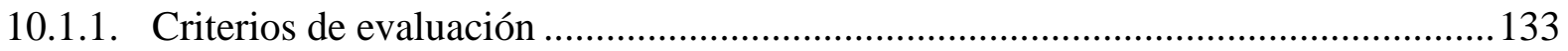

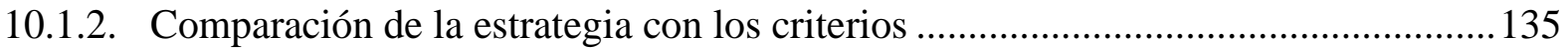

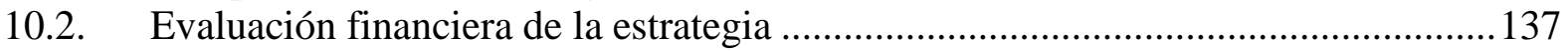

10.2.1. Proyección de estados financieros (situación actual y con la nueva estrategia)...... 140

10.2.2. Estado de resultados (situación actual y con la nueva estrategia) .......................... 150

10.2.3. Balance General (situación actual y con la nueva estrategia)................................151

10.2.4. Flujo de efectivo (situación actual y con la nueva estrategia) ................................ 153

10.2.5. Proyección de flujos (situación actual y con la nueva estrategia) ...........................156

10.2.5.1.Proyección de Flujo de Caja Proyectado sin Estrategia ........................................ 156

10.2.5.2. Proyección de Flujo de Caja Proyectado con Estrategia ....................................... 157

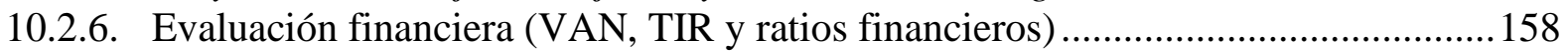

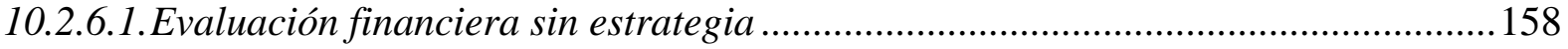

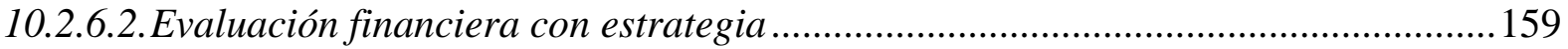

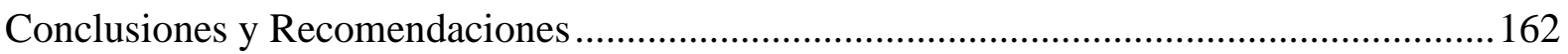

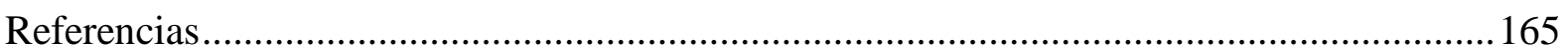

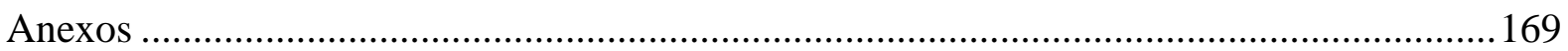




\section{Lista de Tablas}

Tabla 1 Perú: Evolución del PBI del periodo 2017/2016, 2018/2017 28

Tabla 2 Manufactura: Valor Agregado Bruto 29

Tabla 3 Perú: Evolución del PBI del periodo 2016/2015, 2017/2016 30

Tabla 4 Manufactura: Valor Agregado Bruto 31

Tabla 5 Matriz de la visión propuesta 32

Tabla $6 \quad$ Matriz de la misión propuesta 33

Tabla 7 Alineación de la visión con los valores 36

Tabla $8 \quad$ Alineación de la misión con los valores 36

Tabla 9 Impacto en clientes / proveedores de cada una de las variables del entorno $\quad 47$

Tabla 10 Efecto en la empresa de cada una de las variables del entorno 48

Tabla 11 Oportunidades $\quad 49$

Tabla 12 Amenazas $\quad 49$

Tabla 13 Matriz de evaluación de los factores externos (EFE) 50

Tabla 14 Principales empresas del mercado $\quad 56$

Tabla 15 Resumen de la Empresa "Corporación Gráfica Navarrete S.A." 56

Tabla 16 Resumen de la Empresa "Tai Loy S.A." 56

Tabla 17 Resumen de la Empresa "Vivalo Import Export E.I.R.L." 57

$\begin{array}{lll}\text { Tabla } 18 & \text { Productos sustitutos } & 67\end{array}$

Tabla 19 Nuevos competidores $\quad 67$

Tabla 20 Poder de Negociación de los clientes $\quad 68$

Tabla 21 Poder de Negociación de los proveedores $\quad 69$

$\begin{array}{ll}\text { Tabla } 22 \text { Rivalidad entre competidores } & 70\end{array}$

Tabla 23 Análisis del Grado de atractividad de la industria 71

Tabla 24 Propuesta de Factores Críticos de Éxito para la MPC de Vivalo Import Export E.I.R.L.

Tabla 25 Matriz de Perfil Competitivo de la empresa Vivalo Import Export E.I.R.L. $\quad 73$

Tabla 26 Cultura Organizacional de Vivalo Import Export E.I.R.L. 75

Tabla 27 Indicadores Financieros $\quad 88$

Tabla 28 Comparación con el líder de la industria 89

Tabla 29 Matriz VRIO de la empresa Vivalo Import Export E.I.R.L. 91

Tabla 30 Ventajas competitivas de la empresa 92

Tabla 31 Matriz EFI de la empresa Vivalo Import Export E.I.R.L. 95

Tabla 32 Objetivo Estratégico 01

Tabla 33 Objetivo Estratégico $02 \quad 98$

Tabla 34 La estrategia del océano rojo frente a la estrategia del océano azul 100

Tabla 35 Los seis principios de la estrategia del océano azul 100

Tabla 36 Estado actual de los factores claves de la industria $\quad 101$

Tabla 37 Preguntas para crear una nueva curva de valor $\quad 103$

Tabla 38 Principios de las nuevas estrategias $\quad 104$

Tabla 39 Matriz FODA de la empresa Vivalo Import Export E.I.R.L. 107 
Tabla 40 Matriz PEYEA de la empresa Vivalo Import Export E.I.R.L. 108

Tabla 41 Participación de mercado de los principales competidores en soles 112

Tabla 42 Participación de mercado de los principales competidores en porcentaje (\%) $\quad 112$

Tabla 43 Participación de ingresos y utilidades por unidad de negocios (Año 2018) 112

Tabla 44 Tasa de crecimiento de los productos al año $2018 \quad 113$

Tabla 45 Determinación de los datos para la matriz BCG (al 31/12/2018) 113

Tabla 46 Resumen de las estrategias formuladas de la empresa Vivalo Import Export E.I.R.L.

Tabla 47 Matriz de Decisión Estratégica (MDE) de Vivalo Import Export E.I.R.L. 118

Tabla 48 Matriz de escenarios $\quad 119$

Tabla 49 Comparación de estrategias con escenarios de la empresa Vivalo Import 120

Tabla 50 Matriz MPEC de la empresa de la empresa Vivalo Import Export E.I.R.L. 122

Tabla 51 Matriz de Indicadores de los objetivos específicos 126

Tabla 52 Matriz de Iniciativas Estratégicas $\quad 127$

Tabla 53 Matriz de objetivos, indicadores e iniciativas de las estrategias $\quad 129$

Tabla 54 Matriz de presupuesto de las iniciativas estratégicas $\quad 130$

Tabla 55 Matriz de cronograma de las Iniciativas Estratégicas $\quad 131$

Tabla 56 Matriz Rumelt de Vivalo Import Export E.I.R.L. 136

$\begin{array}{llr}\text { Tabla } 57 & \text { Beta apalancado } & 138\end{array}$

Tabla 58 Riesgo país de Perú $\quad 139$

Tabla 59 Obligaciones Financieras al 31-12-2018 140

Tabla 60 Estado de Situación Financiera Histórico (Años 2015 - 2018) 141

Tabla 61 Estado de Resultados (Situación actual) 142 Tabla 62 Ratios Financieros de la Empresa Vivalo Import Export E.I.R.L. (Años 2015144

$\begin{array}{llr}\text { Tabla } 63 & \text { Proyección de precios de venta } & 146 \\ \text { Tabla } 64 & \text { Proyección de costos unitarios } & 147\end{array}$

Tabla 65 Nivel de producción $\quad 147$

Tabla 66 Lanzamiento de coleccionables (Figuritas) 148

Tabla 67 Programa de Inversión con la estrategia 148

Tabla 68 Gastos de Administración (Sin Estrategia) 149

Tabla 69 Gastos Administrativos (Con Estrategia) 149

Tabla 70 Gastos de Venta (Sin Estrategia) 149

Tabla 71 Gastos de Venta (Con Estrategia) 149

Tabla 72 Proyección de los Ingresos con Estrategia 150

Tabla 73 Estado de Resultados de la empresa Vivalo Import Export E.I.R.L. con la 150

Tabla 74 Balance General de la Empresa Vivalo Import Export E.I.R.L. con la 152

Tabla 75 Flujo de Efectivo (Situación Actual) 154

Tabla 76 Flujo de Efectivo (con la Nueva Estrategia) 155 
Tabla 77 Flujo de Caja Proyectado situación actual (sin estrategia) 156

Tabla 78 Flujo de caja proyectado con estrategia 157

Tabla 79 Evaluación del Flujo de Caja sin la Estrategia 158

Tabla 80 Evaluación del Flujo de Caja con la estrategia 159

Tabla 81 Flujo de Caja Incremental con la Estrategia 159

Tabla 82 Ratios financieros con la Estrategia 160 


\section{Lista de Figuras}

Figura 1. Ilustraciones de colecciones de álbumes y figuritas. 22

Figura 2. Ilustraciones de colecciones comercializadas en el rubro de Cards 22

Figura 3. Ilustraciones de colecciones comercializadas en el rubro de Postales 23

Figura 4. Ilustraciones de colecciones comercializadas en el rubro de muñecos de $\begin{array}{ll}\text { colección } & 23\end{array}$

Figura 5. Etapa de madurez de los álbumes y figuritas 25

Figura 6. Etapa de crecimiento de los cards coleccionables 26

Figura 7. Etapa de introducción de las postales y muñecos de colección 26

Figura 8. Organigrama de la empresa Vivalo Import Export E.I.R.L. 28

Figura 9. Producto Bruto Interno y Demanda Interna 2008_I-2018_II (Variación porcentual del índice de volumen físico respecto al mismo periodo del año anterior) Año Base 2007=100. 40

Figura 10. Perú: Evolución del ingreso real promedio per cápita mensual, 2007-2017 (Soles constantes base=2017 a precios Lima Metropolitana. 42

Figura 11: Participación en el mercado de las principales empresas 57

Figura 12. Las cinco fuerzas que moldean la competencia en un sector. 59

Figura 13. Actividades de la cadena de valor de la empresa. $\quad 84$

Figura 14. Modelo Analítico para la Formación de la Estrategia. 98

Figura 15. Lienzo de la Estrategia de la Empresa Vivalo Import Export E.I.R.L. 102

Figura 16. Lienzo de la estrategia actual de la industria. 103

Figura 17. Lienzo de la nueva estrategia. 105

Figura 18. Estrategia por desarrollar según el resultado del Eje X y Y de la Matriz PEYEA de la empresa Vivalo Import Export E.I.R.L. 109

Figura 19. Matriz Interna Externa de la empresa Vivalo Import Export E.I.R.L. $\quad 110$

Figura 20. Matriz Boston Consulting Group (BCG) de la empresa Vivalo Import $\begin{array}{ll}\text { Export E.I.R.L. } & 114\end{array}$

Figura 21. Matriz de la Gran Estrategia de la empresa Vivalo Import Export E.I.R.L. 115

Figura 22. Mapa Estratégico de la empresa Vivalo Import Export E.I.R.L. 124

Figura 23. Ventas vs utilidad neta de la empresa Vivalo Import Export E.I.R.L. 143

Figura 24. Ciclo de conversión de efectivo 145

$\begin{array}{ll}\text { Figura 25. Margen neto vs ROE } & 145\end{array}$ 


\section{Lista de Anexos}

$\begin{array}{lll}\text { Anexo 1. Entrevista a la gerente general } & 170\end{array}$

$\begin{array}{lll}\text { Anexo 2. } & \text { Entrevista al gerente comercial } & 178\end{array}$

Anexo 3. Entrevista al coordinador de administración 185

Anexo 4. Entrevista al coordinador de producción 193

Anexo 5. Entrevista al asistente de despacho 200

Anexo 6. Entrevista al vendedor 203

Anexo 7. Entrevista a cliente (distribuidor) 206

$\begin{array}{lll}\text { Anexo 8. Entrevista a proveedor } \mathrm{N}^{\circ} 01 & 211\end{array}$

$\begin{array}{lll}\text { Anexo 9. } & \text { Entrevista a proveedor } \mathrm{N}^{\circ} 02 & 214\end{array}$

Anexo 10. Entrevista Experto 01

Anexo 11. Entrevista Experto $02 \quad 227$

Anexo 12. Entrevista Experto 03

Anexo 13. Guía de pautas de Focus Group 238

Anexo 14. Transcripción de Focus Group 240 